\title{
LA LÓGICA ENTRE LAS MATEMÁTICAS Y EL DERECHO \\ Preguntas a partir de un intento de analogía
}

\begin{abstract}
ALEJANDRO MARTÍN MALDONADO
As in mathematics, in law it is fundamental to be able to show the reasons why a judicial opinion should be accepted. Assuming this analogy between both disciplines, the article analyzes different conceptions of argumentative logic in mathematics, takes this reflection to the field of law, and holds that in both fields axioms or principles are not chosen because they are self-evident truths, but that this choice is in great measure determined by their implications. Finally, the author asks about the specificity of each of these disciplines, and the point at which the analogy between them no longer holds.
\end{abstract}

Para introducir esta ponencia es necesario que comience por situarme, por presentarme ${ }^{1}$. Estudié matemáticas en la universidad y desde muy pronto comencé a hacerme preguntas acerca de las matemáticas. iDe dónde proviene ese carácter de certeza absoluta que les atribuimos? ¿Qué tipo de objetos son esos con los que tratamos? La clase que, en la carrera de matemáticas, parecía que debería ocuparse de tales cuestiones, era la clase de lógica. Sin embargo, la clase de lógica muy pronto se centraba en una serie de sofisticadas técnicas matemáticas que, al menos para alguien que estaba comenzando, hacían muy difícil entender

\footnotetext{
${ }^{1}$. Este escrito procede, en buena parte, de las muchas conversaciones que hemos tenido con el decano de la Facultad de Derecho y Ciencias Sociales de Icesi; en especial, de aquellas en las que hemos intentado juntar temas aparentemente disímiles como matemáticas, derecho y filosofía (y tantas otras cosas más). Los comentarios de Aquiles Arrieta y Mauricio Rengifo acerca de lógica y derecho fueron determinantes a la hora de pensar la manera de diseñar este texto. Con Magdalena Holguín hemos compartido siempre la necesidad de sacar la reflexión filosófica de las cuatro paredes en las que suele estar encerrada; debo agradecerle muy especialmente la lectura cuidadosa de este texto y sus indicaciones para que mi ignorancia del derecho no fuese tan evidente. Sin embargo, yo soy el único responsable de todas las imprecisiones y exageraciones.
} 
cómo respondían esas preguntas originales. Me di cuenta, entonces, de lo siguiente: las preguntas que me estaba haciendo eran filosóficas y pensé que quizás a quien debería pedir asistencia era a los expertos en esa rama. Comencé a tomar cursos de filosofía y a leer a los filósofos buscando las respuestas que ellos me pudieran sugerir. Aprendí mucho, pero también pude notar que la mayoría de esos filósofos miraban las matemáticas desde muy lejos. Me encontré así en una encrucijada de la cuál aún no se muy bien cómo salir: por un lado los matemáticos suelen despreciar las discusiones si no logran formalizarlas (es decir, convertirlas en matemáticas) y, por otro, muchos filósofos pretenden hablar de las matemáticas sin adentrarse en ellas más de lo que les exigió el colegio².

¿Pero cómo se relaciona todo esto con el derecho que es el tema que aquí nos interesa? Resulta que, buscándome un lugar en esa encrucijada, encontré que se podía intentar que la lógica no fuese únicamente esa disciplina técnica que desarrollan los matemáticos, sino que se aprovecharan sus resultados para hacer una reflexión en la que el lenguaje no fuese exclusivamente para iniciados. La lógica, tal como nos suena a la mayoría, aquella disciplina que se ocupa de los argumentos y de los razonamientos, tiene interés tanto para las matemáticas como para la filosofía, pero también para las distintas ciencias (física, biología, de manera muy particular en medicina) y, por supuesto, para el derecho. Es quizás en esta última donde la crítica de argumentos ocupa un lugar tan fundamental como en la matemáticaª

\footnotetext{
${ }^{2}$ Finalmente, gracias a mi profesor Xavier Caicedo, hice una tesis sobre la lógica de Leibniz, donde pude entrecruzar mis intereses matemáticos y filosóficos. Para mí, Leibniz ha sido desde entonces un motivo muy especial de búsqueda y una referencia inspiradora acerca de los puentes que la lógica permite tender. Abogado de formación, Leibniz (1646-1716) es famoso por su interés en una revitalización de la lógica (Característica Universal) y sus investigaciones matemático-filosóficas (Cálculo diferencial, Monadología). Sus escritos jurídicos son un ejemplo muy interesante de lo que puede aportar la perspectiva del lógico al análisis del derecho (Escritos de filosofía política y jurídica, Biblioteca nueva, Madrid 2001). Por un lado, Leibniz busca una fundamentación del derecho basada en definiciones, y por otro, trabaja en una teoría jurídica en la que la filosofía del lenguaje (y en particular la gramática) juegan un papel muy importante. Con respecto al tema que vamos a tratar resulta pertinente resaltar la analogía que encuentra entre los conectivos modales (necesario/posible) y los deónticos (obligatorio/permitido) con el fin de desarrollar un cálculo lógico para el derecho.

${ }^{3}$ La definición de la lógica como crítica de argumentos se la debo al filósofo norteamericano Charles Sanders Peirce (1839-1914). Peirce debería estar junto con Aristóteles, Leibniz y Frege como uno de los más grandes lógicos de la historia. Simultáneamente con el último, llevó a cabo toda una revolución en la disciplina que amplió infinitamente su horizonte de aplicación. Peirce hizo parte, junto con el Juez Holmes y William James, del llamado "club de los metafísicos", reunión de intelectuales de Boston de la que se dice surgió el pragmatismo americano. Esta corriente filosófica nace del esfuerzo por resaltar el lugar que juega la práctica en nuestras concepciones. Peirce presentó ante el club su ponencia La fijación de la creencia donde hay una teoría del razonamiento en la que la relación acción-creencia juega un papel determinante. Ese texto habrá de considerarse
} 
Tanto en derecho como en matemáticas (podríamos incluir aquí también la filosofía), las tesis se sostienen casi exclusivamente a partir de argumentos. En física, los experimentos tienen un lugar clave para establecer la verdad de las teorías; en medicina, si bien razonar correctamente es fundamental, todo termina midiéndose con la práctica. En cambio, en matemáticas un teorema no se verifica experimentalmente, es preciso demostrarlo. Y la lógica, precisamente, ha de investigar los criterios para juzgar una demostración y las implicaciones que tiene aceptar ciertas cosas como demostraciones y negar otras. En derecho, toda afirmación reclama un argumento, que no suele llamarse demostración, pero al que se le exige nos muestre la razón por la cual hemos de aceptar un veredicto o una decisión.

El objeto de análisis aquí es la analogía que se ha hecho entre derecho y matemáticas con respecto a su estructura lógica. Toda analogía tiene tres elementos: los dos extremos y la relación (proporción) que se encuentra entre ellos. Comenzaré por examinar la analogía clásica, basada en una concepción de la matemática que explica la verdad matemática mediante el esquema axiomas-teoremas. El derecho funcionaría análogamente de manera exclusivamente deductiva. Señalaré las preguntas que han surgido y pueden surgir de considerar que esa es la estructura lógica del derecho. Luego cuestionaré esa concepción superficial de la matemática, señalando un par de ejemplos que nos permitan aproximarnos a una visión que describa mejor la práctica y la "lógica" de las matemáticas ${ }^{4}$. A partir de allí plantearé unas cuestiones que surgirían de intentar llevar una reflexión análoga en el caso del derecho. Para terminar

como la piedra fundacional del pragmatismo. Luis Menand en su libro El Club de los Metafísicos (Destino, Barcelona 2001), describe muy finamente el contraste entre los diferentes pensadores que allí se reunían. Para nosotros será muy interesante el análisis que hace de la manera como está implicada la filosofía pragmatista en la teoría jurídica que, más adelante, el Juez Holmes esarrollaría. ${ }^{4}$ El impulso para cuestionar esta concepción de la verdad matemática lo tomo de las reflexiones en filosofía de la matemática de Gian-Carlo Rota (1932-1999). Profesor de MIT y especialista en combinatoria, Rota era también un filósofo tremendamente agudo. Fue famoso por la polémica que mantuvo con los que regían en los departamentos de filosofía. Su artículo "La perniciosa influencia de la matemática en la filosofía" constituye una ácida crítica de la filosofía analítica, donde los acusa de abusar del lenguaje matemático para aparentar rigor, pero alejándose de los verdaderos problemas filosóficos. Este artículo mío habría podido ser algo análogo, reemplazando "filosofía" por "derecho", pero pretendo ir más allá aprovechando la propia reflexión de Rota sobre las matemáticas. Él toma como punto de partida la fenomenología de Husserl para hacer un análisis detallado de lo que realmente sucede en matemáticas. Son sus fenomenologías de la verdad y de la demostración en matemáticas las que me guían para intentar que la matemática sea una provechosa influencia a la hora de reflexionar sobre el derecho. Le debo al lógico-matemático Andrés Villaveces haberme introducido en el estudio de Rota. Con él estamos ahora traduciendo sus textos e intentando seguir sus pistas para pensar acerca de las matemáticas. 
daré algunos indicios que nos lleven a reconocer los límites de la analogía que se intenta establecer entre matemáticas y derecho, dadas las peculiaridades de cada uno de los extremos.

\section{Explicación formal de la verdad matemática.}

La forma tradicional como suele explicarse la verdad de los enunciados matemáticos es mediante el esquema: axiomas-teoremas. Demostrar un teorema consiste en señalar como se deduce la proposición enunciada a partir de los axiomas y definiciones de la teoría. Además, cuando una proposición se deduce de otras, la verdad de las primeras implica la verdad de la proposición deducida. En este caso, la verdad del enunciado del teorema se heredaría de la verdad de los axiomas. Por lo tanto, si pretendemos que una demostración prueba que el enunciado es verdadero, deberíamos entonces suponer que los axiomas lo son. Pero ison verdaderos los axiomas?

Dado nuestro esquema no cabría demostrar los axiomas, ya que ellos están a la cabeza del sistema. Por lo tanto la verdad de los axiomas debería ser de un orden distinto a la de los teoremas. Hay dos opciones clásicas:

1. Los axiomas son verdades evidentes.

2. Los axiomas son convenciones.

La primera parece aún tener mucha aceptación y no es raro incluso escuchar a matemáticos defendiendo tal posición. La segunda es más popular entre los filósofos, aunque cada vez son más los matemáticos, especialmente los que son afines a la lógica, quienes estarían inclinados a sostenerla.

Al considerar seriamente aquella afirmación según la cual los axiomas de la matemática constituyen verdades evidentes, muy pronto surgen preguntas de este tipo: ¿En qué sentido evidentes? ¿Dónde miramos para ver la verdad de estos axiomas? ¿De qué forma ellos muestran su propia verdad?

Dos ejemplos de teorías matemáticas que pueden servirnos para ilustrar estos problemas son la geometría y la aritmética. La primera es la más propia para hablar con términos "visuales" como lo implica el lenguaje de la evidencia. La segunda, en cambio, trata de números, ítems con los cuales nos queda más difícil decir que tengamos una relación perceptual directa.

La geometría fue durante siglos el modelo para toda ciencia que quisiese ser demostrativa, tanto así que, cuando se escribía de esa forma, se hablaba 
de more geometrico (la Ética de Spinoza uno de los ejemplos más particulares de la tentación de escribir de esta forma). Precisamente uno de los hechos que marcó la historia de la geometría fue la reflexión alrededor de uno de sus axiomas (que Euclides llamó postulados, escogencia terminológica acerca de la cual podremos reflexionar un poco más adelante). El famoso quinto postulado, llamado "de las paralelas" dice lo siguiente:

Dadas dos rectas que se intersecan con una misma recta, si los ángulos internos suman menos que dos rectos, estas dos rectas se cruzarán de ese lado.

¿Se trata de una verdad evidente? ¿En qué sentido? Alguien podría preguntarse iSi la suma de los ángulos es casi $180^{\circ}$ cómo sé yo que las rectas se han de cruzar? Preguntas como ésta, y el hecho de que el enunciado del quinto postulado fuese tan diferente de los anteriores hizo que muchos matemáticos, de muy distintas épocas, se preguntaran si no se trataría más bien de un teorema. Esta cuestión no pudo ser resuelta sino hasta el siglo XIX, cuando fue demostrado que era imposible probar (o refutar) el quinto a partir de los otros cuatro. Lo interesante, y novedoso, de este resultado es que ya no demuestra una propiedad de los objetos matemáticos, sino de las teorías. La estrategia de esta prueba consiste en mostrar que existen estructuras (modelos) que pueden satisfacer los primeros cuatro postulados, pero no el quinto. Estas son las famosas geometrías no-euclídeas. Su existencia pone en duda la "evidencia" de las proposiciones de la geometría euclídea. Se llegaría entonces incluso a afirmar que la geometría "real" del espacio físico podría ser no-euclídea (Einstein).

Consideremos ahora la aritmética. En la base están los resultados de los cálculos elementales (las sumas y multiplicaciones que todos conocemos), y de allí en adelante todo tipo de teoremas generales acerca de los números. Desde sus orígenes pitagóricos se conocen muchos de estos resultados (como el hecho de que la suma de los impares consecutivos da siempre un número cuadrado $^{5}$ ), pero sólo a finales del siglo XIX se presenta un sistema axiomático (Peano). ¿Cómo se demostraban antes las proposiciones? ¿Qué garantizaba su verdad? Incluso, asumiendo que los axiomas eran "implícitos", ahora que son explícitos: ien qué sentido se puede decir que son evidentes? ¿En dónde se mira ahora para ver si valen o no para los números?

$$
\begin{aligned}
& 51+3=4=2^{2} \\
& 1+3+5=9=3^{2} \\
& 1+3+5+7=16=4^{2}
\end{aligned}
$$


Una solución que podría adoptarse consistiría en afirmar que los axiomas son convenciones que se fijan en algún momento. Esto eliminaría la pregunta por la "evidencia" de los mismos, pero abriría cuestiones nuevas: ¿Si son convencionales, entonces toda la matemática resultaría arbitraria? Sin embargo, como hemos visto, los axiomas de la aritmética sólo aparecen cuando esta disciplina está ya bien madura: iLa convención estaba implícita antes en el trabajo de los matemáticos?

\section{Preguntas para el derecho.}

Para el caso del derecho me voy a limitar a plantear unas preguntas que mis compañeros que conocen mucho mejor el tema me pueden ayudar a responder. Dada la estructura jerárquica de los enunciados en el derecho, podría pensarse en una serie de analogías suponiendo que la deducción jugase un papel importante en la argumentación jurídica. Tendríamos que buscar qué correspondería al par axioma/teorema y qué ocuparía el lugar de la deducción, que es el camino que en matemática lleva de los primeros a los segundos.

El par axioma/teorema podría corresponder en teoría del derecho a la pareja principios/leyes o en la práctica jurídica a la manera como se argumenta en un caso a partir de los códigos. El primer intento de analogía lleva a encontrarnos en la teoría del derecho preguntas muy parecidas a las que se han dado en la filosofía de la matemática. Si se dice que la validez de las leyes depende de la de unos ciertos principios generales, somos llevados a preguntarnos: ¿Qué sostiene entonces los "principios del derecho"? ¿Son intuitivamente verdaderos? iSon verdades "naturales"? ¿Son meras convenciones sociales?

El segundo intento de analogía nos obliga a precisar un poco más los términos. En el par leyes y normas/decisiones jurídicas: ¿En qué sentido puede ser una decisión judicial particular análoga a un teorema matemático? ¿No es particular la primera, mientras que el segundo se trata de un resultado general? Esto nos lleva a reflexionar acerca de la distinción general/particular que no resulta ser tan tajante como lo parece a primera vista. En muchos de los casos, los teoremas matemáticos surgen luego de intentar buscar una respuesta para un caso particular. Sin embargo, una vez resuelto ese problema se encuentra en qué medida se puede usar un argumento del mismo tipo para casos similares y se obtiene un resultado con cierta generalidad, pero la esencia del teorema ya estaba en la prueba para el caso particular. En derecho, ya sea en un caso de la decisión de un juez o de las cortes (constitucional o suprema) finalmente debe buscarse una nor- 
ma que se siga de las leyes y normas establecidas que cobije el caso en cuestión. Dado que la pregunta por el "fundamento" de las leyes pertenece más bien a lo que examinamos más atrás, aquí lo que nos interesa es la manera como estarían conectadas las normas a las que se apela en la decisión con las leyes y normas establecidas. ¿Se trata efectivamente de una deducción? ¿Debe buscarse un formalismo jurídico, como el que se desarrolló para las matemáticas, para garantizar el rigor de las deducciones? Ese formalismo, "la lógica del derecho" que regularía las demostraciones, iserá el mismo que para las matemáticas?

\section{Otra visión de la verdad en matemáticas.}

Los resultados del siglo XIX en matemáticas pueden leerse de tal manera que nos hagan mirar de manera distinta la forma como los matemáticos entienden la verdad de sus enunciados y su relación con la demostración. En el caso de la aritmética, por ejemplo, hay resultados que se consideran verdaderos independientemente de si pueden ser demostrados por cierto sistema axiomático o no. Justamente el hecho de que un sistema axiomático sea el resultado de un largo proceso nos viene a confirmar el hecho de que la escogencia de los axiomas no es producto de una especie de iluminación mística. Los axiomas van siendo evaluados hasta encontrar los que mejor se acomoden a las necesidades dadas por una práctica matemática que ya lleva cientos de años produciendo resultados. Entonces, icómo se escogen los axiomas? ¿cómo se los juzga? Investigando qué teoremas se pueden deducir de ellos y cuáles no. No son el resultado de una simple inspección que decida si son evidentes o no, ni el producto del arbitrio de cierto matemático poderoso, o de una comunidad con gran poder de decisión. Se llega a los axiomas después de un lento camino de prueba, de ensayo y error, y sobre todo, de ardua evaluación.

Los axiomas se miden por sus implicaciones. Resultados que ya han sido confirmados por muchos caminos distintos, ahora ponen a prueba a los candidatos a axiomas. Además, no es suficiente encontrar axiomas a partir de los cuales se puede deducir los resultados establecidos. Se requiere también que en la demostración del teorema se haga ver la razón de su verdad. Por lo tanto se buscarán axiomas que, en alguna medida, expresen de la forma más sencilla la esencia del concepto matemático en cuestión. Hay una tensión muy interesante entre los dos criterios para "evaluar" los candidatos a axiomas: (1) sus implicaciones, (2) lo que ellos mismos directamente expresan. 
La geometría euclídea da la sensación de ser simplemente una disciplina que consiste en extraer conclusiones de unos postulados. Pero los postulados no estaban fijos desde el comienzo. El trabajo de Euclides consistió justamente en organizar de esa manera la geometría (axiomático-deductiva). Se considera que para el momento en que Euclides escribió Los Elementos la mayoría de resultados ya habían sido demostrados con anterioridad. Sin embargo, la tarea de Euclides no es para nada despreciable, más bien titánica, ya que consiguió compilar y unificar de una manera muy particular todo el conocimiento geométrico de la época. Uno de los aspectos más interesantes de esa tarea consiste precisamente en encontrar un mínimo de proposiciones a partir de las cuales se puedan seguir todas las demás. Estos elementos básicos vienen a ser las definiciones, las nociones comunes y los postulados, que como su nombre lo dice, no pretenden ser más que proposiciones que postulan la posibilidad de trazar ciertos objetos y la existencia de ciertas relaciones entre algunos de ellos. En las demostraciones de los Elementos no sólo vemos cómo se pueden probar todas las proposiciones a partir de estos "elementos", sino que entendemos por qué éstas son verdaderas.

Este es un punto importante en el que quisiera detenerme. Esta propiedad de "hacer ver la verdad" de un enunciado no es algo que compartan todas las demostraciones matemáticas, es más, a veces resulta algo más bien raro. Sin embargo es algo que los matemáticos persiguen asiduamente. Si los matemáticos sólo quisieran demostrar los teoremas, sin preocuparse por este "mostrar su verdad" no se daría un fenómeno en apariencia extraño pero recurrente en la historia de las matemáticas: los teoremas son probados una y otra vez pasando de intrincados argumentos que sólo se pueden seguir paso a paso, hasta llegar a pruebas cada vez más sencillas ${ }^{6}$.

\section{Preguntas para el derecho.}

Ya vimos que en el derecho el par axioma/teorema puede corresponder a cosas diferentes en el derecho. En el caso de la pregunta por los "principios del derecho" deberíamos notar que en matemáticas los "principios" (los axiomas) no son en caso alguno el fundamento, sino que más bien dependen de los teoremas. Sin embargo los axiomas siguen jugando un papel clave en la organización de las matemáticas, en la forma como han de ser clarificados sus conceptos y presentados sus resultados. iLos principios del derecho tienen un interés que vaya más allá de una búsqueda de fundamentación?

\footnotetext{
${ }^{6}$ Gian-Carlo Rota en "La fenomenología de la demostración matemática." (Indiscrete Thoughts. Birkhäuser, NY 1997) da cuenta, detalladamente y mediante varios ejemplos, de este fenómeno.
} 
Pero me interesa más aquí ver como la reflexión acerca de las matemáticas nos invita a pensar la práctica cotidiana del derecho: la manera como se escogen las leyes, como se toman las decisiones jurídicas, como se relacionan las leyes y normas escritas con la práctica. ¿Qué está antes: la ley o sus consecuencias? Hemos descrito para las matemáticas una dinámica que va de los teoremas a los axiomas, donde primero se obtienen unos resultados particulares y luego se va articulando una teoría formal. En derecho sería interesante analizar también este tipo de dinámica; estudiar la forma en que las decisiones de los jueces y de las cortes pueden ir determinado las leyes, o incluso, la forma como las leyes pueden ser entendidas a partir de sus consecuencias prácticas.

Están en juego aquí dos tipos de consideración, uno más bien descriptivo que tiene que ver con mirar efectivamente la manera como está articulada la trama argumentativa jurídica, y otro, quizás normativo, que se pregunta cuál sería la mejor manera de argumentar en el derecho. Es interesante mirar también cómo ciertas concepciones de la ley, y de la argumentación jurídica (en principio descriptiva), han inspirado sistemas jurídicos (normativos). Entonces, al hacer el análisis de la argumentación jurídica no sólo tenemos que mirar la forma como funcionan esos sistemas sino la idea de ley y de derecho que los inspira (podría haber una gran distancia entre la teoría normativa que inspira un cierto sistema jurídico y la práctica dentro del mismo).

Tenemos que preguntarnos entonces: ¿Cómo argumentan los jueces? En un sistema como el nuestro con un código claramente establecido, iqué conexión lógica existe entre las leyes y los veredictos? ¿Y entre las leyes y las normas que regulan el comportamiento de los ciudadanos? ¿En qué casos el código no puede decidir? Con respecto a esta última pregunta se ha debatido mucho últimamente en Colombia, en especial acerca del papel que juega la Corte Constitucional. Por un lado, ella debe vigilar que los proyectos de ley sean acordes con la constitución: ¿qué tipo de relación lógica es "ser acorde"? ies lo mismo que "consistente"? Y por otro, la Corte toma decisiones con respecto a las tutelas que, en principio, tratan de casos particulares. Allí vemos un ejemplo concreto de cómo los casos particulares no lo son tanto, ya que se convierten en precedentes que sirven para decidir casos posteriores. Estos precedentes en alguna manera dejan de ser particulares para convertirse en esquemas o paradigmas que van a jugar un papel muy importante en las decisiones legales futuras.

Venía diciendo que para el caso de las matemáticas entender un axioma en gran medida es entender sus implicaciones. Uno puede "creer que en- 
tiende" una proposición porque entiende lo que significa cada una de las palabras que aparecen en ella y la forma como están conectados. Pero luego se da cuenta, al examinar sus consecuencias, que la comprensión era muy superficial. En el derecho esta relación es quizás más patente. Una ley puede parecer la más justa, la más adecuada, sin embargo puede tener consecuencias que la hagan inmanejable, o incluso, indeseable. Esta relación entre las leyes y sus consecuencias y el peso que se quiera dar a los distintos lados de la balanza implican concepciones del derecho muy diferentes ${ }^{7}$. Para mí es interesante mirar qué efectos tiene dar más importancia a un lado o a otro y las distintas lógicas (formas de pensar y argumentar) que están implicadas en las diferentes prácticas del derecho que una u otra decisión implican.

\section{Diferencias entre las matemáticas y el derecho}

Hasta ahora me he concentrado en señalar las preguntas que pueden hacérsele al derecho si suponemos una cierta analogía con las matemáticas. Ahora quisiera indicar brevemente algunas preguntas que pueden aparecer al poner en cuestión tal analogía. O sugerir al menos ciertas pistas para pensar lo específico de cada una de las dos disciplinas, lo que las diferencia, al menos con respecto a las cuestiones lógicas.

Uno de los aspectos que más se ha subrayado y que ha tendido a llevar a los desarrollos más sofisticados dentro de la lógica matemática aplicada al derecho es el papel que juega en ella la contradicción. En matemáticas, suele decirse (lo que a veces es una exageración) que encontrar una contradicción en una teoría es fatal para la misma. Según la lógica clásica, de una contradicción se sigue cualquier cosa y, por lo tanto, una teoría que implicara una contradicción se vaciaría de sentido. Sin llegar a tales extremos, lo que sí podemos decir es que en matemáticas se busca siempre evitar la contradicción; lo que se quiere son teorías consistentes, y si bien una contradicción nunca es una tragedia, en caso de encontrarla, siempre se buscará la manera de reformar la teoría original. Dada la complejidad de los sistemas legales y la dificultad para reformarlos podríamos preguntarnos: iefectivamente hay que evitar a toda costa los sistemas contradictorios? ¿cómo se puede seguir argumentando dentro de ellos sin que esto implique su anulación; que cualquier cosa pueda decirse? Las contra-

\footnotetext{
${ }^{7}$ En el caso colombiano han sido muy interesantes los debates entre la Corte Constitucional y el Banco de la República por un lado, y el Ejecutivo por otro, donde ha sido de capital importancia la relación entre las leyes y sus implicaciones económicas. Incluso muchos dijeron que la motivación principal del referendo eran cuestiones económicas que no se podían comprender simplemente al examinar el enunciado de las propuestas.
} 
dicciones muchas veces aparecen por la ambigüedad de los términos. Pero la ambigüedad es una condición de los términos en el lenguaje ordinario. ¿Debe ésta evitarse a toda costa en el derecho? ¿No hay ambigüedades inevitables? ¿No puede haber incluso ambigüedades provechosas?

En matemáticas, por el contrario, se tiende a buscar siempre un marco en el cual el uso de los signos esté determinado. Para hacer las pruebas particulares es indispensable tener los términos claramente definidos. El hecho de que un mismo concepto atraviese distintos contextos inspira un nuevo contexto donde se pueden hacer pruebas generales. Pero este nuevo contexto ha de tener unas bases claramente establecidas. Podríamos preguntarnos si sucede lo mismo en el derecho, si siempre es necesario tener un marco determinado en términos de códigos, de leyes y definiciones.

Una pregunta muy interesante que surgió cuando conversábamos para armar este seminario fue: ¿dónde tiene más libertad el juez? ien un sistema como el derecho romano o en el anglosajón? Aquí lo que está en juego es en qué medida está determinada la decisión jurídica (ya sea del juez o de las cortes) por los códigos o por los precedentes y qué papel juega quien está tomando la decisión. En matemáticas, una vez fijado un sistema axiomático, el que una proposición se siga o no puede ser establecido con rigor. En principio la personalidad del que demostró un teorema no juega ningún papel, si lo demostró no hay ninguna decisión que tomar. iSucede lo mismo en el derecho? Últimamente se ha subrayado mucho el papel que juega la interpretación en el derecho. El que toma la decisión ha de hacerlo basándose en la interpretación de los códigos y normas. ¿Qué tanta libertad hay a la hora de interpretar? Tal como he presentando la deducción en matemáticas, ella comparte algo esencial con la interpretación ya que finalmente es la que permite dar significado a los términos matemáticos, a las proposiciones. ¿Es radicalmente diferente la interpretación que hacen los jueces de las leyes de la deducción?

Sin embargo, muchos de los casos más interesantes en derecho, y en matemáticas, son aquellos donde la proposición no parece estar determinada por los principios (llámense axiomas o leyes). ¿Cómo argumentar en esos casos en derecho cuando de todas maneras hay que tomar una decisión? ¿Qué hace que en matemáticas a veces se mantengan vivas las dos posibilidades (teorías que compiten) y a veces se decida por un camino particular? A partir de aquí podríamos intentar devolvernos y pensar qué preguntas y respuestas podría sugerir la argumentación jurídica para la reflexión en filosofía de las matemáticas. Pero lo que nos proponíamos aquí era lo contrario, espero que haya quedado tema para el debate. 